\title{
ZEROS OF WEAKLY HOLOMORPHIC MODULAR FORMS OF LEVELS 2 AND 3
}

\author{
SHARON ANNE GARTHWAITE AND PAUL JENKINS
}

\begin{abstract}
Let $M_{k}^{\sharp}(N)$ be the space of weakly holomorphic modular forms for $\Gamma_{0}(N)$ that are holomorphic at all cusps except possibly at $\infty$. We study a canonical basis for $M_{k}^{\sharp}(2)$ and $M_{k}^{\sharp}(3)$ and prove that almost all modular forms in this basis have the property that the majority of their zeros in a fundamental domain lie on a lower boundary arc of the fundamental domain.
\end{abstract}

\section{INTRODUCTION}

In studying a complex-valued function, it is natural to attempt to locate the zeros of the function; in fact, one of the most famous unsolved problems in mathematics asks whether the nontrivial zeros of the Riemann zeta function $\zeta(s)$ lie on a particular line. In this paper, we study the locations of the zeros of certain modular forms, and show that most of their zeros in a fundamental domain occur on a particular circular arc.

For the Eisenstein series, perhaps the easiest examples of modular forms, a great deal is known about the locations of the zeros. In the 1960s, Wohlfahrt [23] showed that for even $4 \leq k \leq 26$, all zeros of the Eisenstein series $E_{k}(z)$ in the standard fundamental domain for $\mathrm{SL}_{2}(\mathbb{Z})$ lie on the unit circle $|z|=1$, and R. A. Rankin [18] extended the range of values of $k$ for which this holds. Shortly afterward, F.K.C. Rankin and Swinnerton-Dyer [17] proved this result for all weights $k \geq 4$. R.A. Rankin [19] also obtained the result for certain Poincaré series, which generalize Eisenstein series. Similar results have been obtained for Eisenstein series for $\Gamma_{0}^{*}(2)$ and $\Gamma_{0}^{*}(3)$ by Miezaki, Nozaki, and Shigezumi [15], for Eisenstein series for $\Gamma_{0}^{*}(5)$ and $\Gamma_{0}^{*}(7)$ and for Poincaré series for $\Gamma_{0}^{*}(2)$ and $\Gamma_{0}^{*}(3)$ by Shigezumi [21, 22], and for a family of Eisenstein series for $\Gamma(2)$ by the first author, Long, Swisher, and Treneer [11].

The above results which locate the zeros of Eisenstein series and Poincaré series use the same general idea of approximating the modular form by an elementary function having the required number of zeros on the arc. For example, the Eisenstein series $E_{k}(z)$ may be written as a sum over an integer lattice. When $z$ is restricted to the unit circle, so that $z=e^{i \theta}$, the four main terms of this series combine to give $2 e^{\frac{-i k \theta}{2}} \cos \left(\frac{k \theta}{2}\right)$. Rankin and Swinnerton-Dyer's proof shows that the additional terms are small, so the zeros of $E_{k}(z)$ are close to the zeros of this trigonometric function.

In 1997, Asai, Kaneko, and Ninomiya [3] used this idea to study the zeros of polynomials related to the modular function $j(\tau)$. The $j(\tau)$ function is a Hauptmodul, or an isomorphism from the quotient of the upper half plane $\mathbb{H}$ under the action of $\mathrm{SL}_{2}(\mathbb{Z})$ to the complex plane $\mathbb{C}$. It generates all modular functions on $\mathrm{SL}_{2}(\mathbb{Z})$, and it also parameterizes the isomorphism classes of elliptic curves over $\mathbb{C}$. The image of $j(z)$ under the Hecke operator $T_{n}$ is a polynomial in $j(z)$, which we write as $P_{n}(j(z))$. Letting $\Delta(z)$ be the modular discriminant, which

2010 Mathematics Subject Classification. 11F11, 11F03. 
is a weight 12 cusp form on $\mathrm{SL}_{2}(z)$ with no zeros in $\mathbb{H}$, a generating function for the $P_{n}(j(z))$ is given by

$$
\sum_{n=0}^{\infty} P_{n}(j(z)) e^{2 \pi i n \tau}=\frac{E_{4}(\tau)^{2} E_{6}(\tau)}{\Delta(\tau)} \cdot \frac{1}{j(\tau)-j(z)} .
$$

Using this generating function, Asai et al. approximated the polynomials $P_{n}(j(z))$ by trigonometric functions well enough to prove that their zeros in the fundamental domain lie on the unit circle.

Duke and the second author [5] extended the results on $j(z)$ to a two-parameter family of modular forms that form bases for spaces of weakly holomorphic modular forms of level 1. In this case, the connection to elementary functions is less direct. Cauchy's integral formula relates the modular forms to a contour integral of a generalized version of the generating function (1.1). An application of the residue theorem produces the elementary functions, and the proof follows by bounding the integral over a range of values for $\tau$ and $z$. The zeros again lie on the unit circle for many of the forms in the family, though in contrast to previous results, it is known that this property does not hold for all of the modular forms.

We mention one further result using a different technique by Hahn [13], who obtained general results on the zeros of Eisenstein series for genus zero Fuchsian groups; the general idea is an analogue of the classical argument that shows that the zeros of an orthogonal polynomial all lie on the real line.

This question of locating zeros of modular forms is made even more interesting by results of Rudnick [20] that showed that the zeros of Hecke eigenforms of weight $k$, in a sense the orthogonal complement of the Eisenstein series, are expected to become equidistributed in the fundamental domain as $k \rightarrow \infty$; this conjecture was proved by Holowinsky and Soundararajan [14] as a consequence of more general work on mass equidistribution for Hecke eigenforms. Ghosh and Sarnak [12] gave a lower bound for the density of zeros lying on certain arcs for such eigenforms. In a different direction, Basraoui and Sebbar [6] proved that the quasi-modular form $E_{2}(\tau)$ has infinitely many zeros that are inequivalent under $\mathrm{SL}_{2}(\mathbb{Z})$, and that none of these lie within the fundamental domain.

In this paper, we examine modular forms in a basis for certain spaces of weakly holomorphic modular forms of arbitrary integral weight and levels 2 and 3 . We show that for almost all of the basis elements, most of their zeros in a fundamental domain for $\Gamma_{0}(2)$ or $\Gamma_{0}(3)$ lie on a circular arc along the lower boundary of the fundamental domain. This is possible because we can again approximate these modular forms by elementary functions; however, the shape of the fundamental domain makes it difficult to accurately locate all of the zeros.

\section{Definitions And Statement of Results}

Let $M_{k}(2)$ be the space of holomorphic modular forms of weight $k$ for the group $\Gamma_{0}(2)=$ $\left\{\left(\begin{array}{ll}a & b \\ c & d\end{array}\right) \in \mathrm{SL}_{2}(\mathbb{Z}): c \equiv 0(\bmod 2)\right\}$, and let $M_{k}^{!}(2)$ be the corresponding space of weakly holomorphic modular forms, or modular forms that are holomorphic on the upper half plane and meromorphic at the cusps. Let $M_{k}^{\sharp}(2)$ be the subspace of $M_{k}^{!}(2)$ consisting of forms which are holomorphic away from the cusp at $\infty$. This space appears, for instance, in [16], where it is shown that traces of negative integral weight forms in such a space appear as coefficients of certain half integral weight forms of level $4 N$. Modular forms in $M_{k}^{\sharp}(2)$ have been studied by Ahlgren [1], who gave explicit formulas for the action of the $\theta$-operator on forms in these spaces and obtained formulas for the exponents of their infinite product expansions, and by 
Andersen and the second author [2], who gave congruences for the coefficients of a basis for $M_{0}^{\sharp}$.

For the group $\Gamma_{0}(2)$, we use a fundamental domain in the upper half plane bounded by the lines $\operatorname{Re}(z)=-\frac{1}{2}$ and $\operatorname{Re}(z)=\frac{1}{2}$ and the circles of radius $\frac{1}{2}$ centered at $z=-\frac{1}{2}$ and $z=\frac{1}{2}$. We include the boundary on the left half of this fundamental domain, which is equivalent to the opposite boundary under the action of the matrices $\left(\begin{array}{ll}1 & 1 \\ 0 & 1\end{array}\right)$ and $\left(\begin{array}{ll}1 & 0 \\ 2 & 1\end{array}\right)$. The cusps of this fundamental domain may be taken to be at $\infty$ and at 0 .

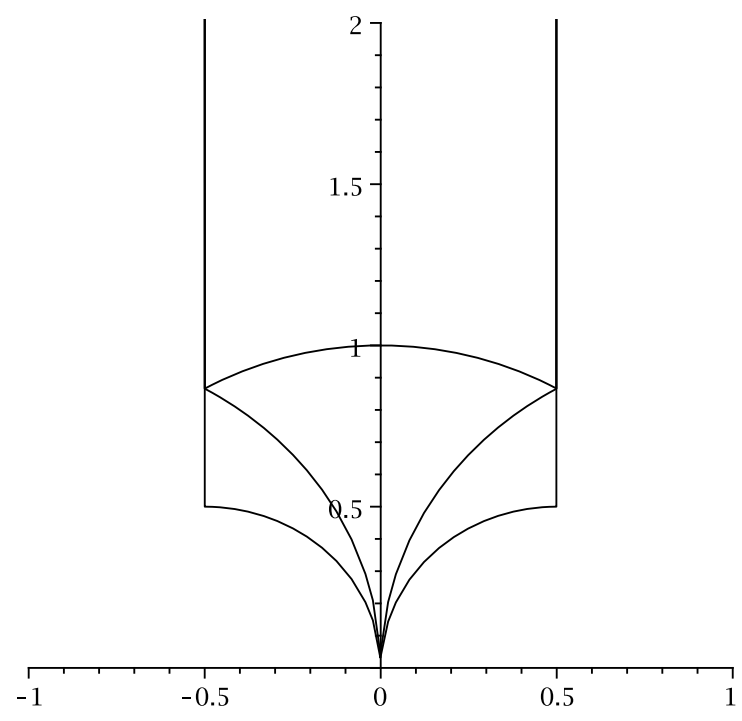

Modular forms in $M_{k}^{!}(2)$ with real coefficients demonstrate a nice property on the lower boundary of this fundamental domain. For a Fourier series $f(z)=\sum a(n) e^{2 \pi i n z}$ with real Fourier coefficients $a(n)$, note that $\overline{f(a+b i)}=\sum \overline{a(n)} e^{-2 \pi i n a} e^{-2 \pi n b}=f(-a+b i)$, or $\overline{f(z)}=$ $f(-\bar{z})$. Thus, for modular forms $f$ of weight $k$ on $\Gamma_{0}(2)$, if we let $z=-\frac{1}{2}+\frac{1}{2} e^{i \theta}$ for $0 \leq \theta \leq \frac{\pi}{2}$, so that $z$ is on the lower boundary of the (symmetric) fundamental domain, we find that $\left(\begin{array}{ll}1 & 0 \\ 2 & 1\end{array}\right) z=\frac{z}{2 z+1}=\frac{1}{2}-\frac{1}{2} e^{-i \theta}=-\bar{z}$. Therefore, $f(z)=(2 z+1)^{-k} f\left(\frac{z}{2 z+1}\right)=e^{-i k \theta} \overline{f(z)}$, and the normalized modular form $e^{\frac{i k \theta}{2}} f\left(-\frac{1}{2}+\frac{1}{2} e^{i \theta}\right)$ is real valued for $\theta$ between 0 and $\frac{\pi}{2}$.

It is useful to define three particular modular forms of level 2. As usual, let $q=e^{2 \pi i z}$. Let

$$
\psi(z)=\left(\frac{\eta(z)}{\eta(2 z)}\right)^{24}=q^{-1}-24+276 q+\ldots \in M_{0}^{\sharp}(2)
$$

be the Hauptmodul for $\Gamma_{0}(2)$. This form has integer coefficients, has a pole at $\infty$, and vanishes at 0 . Moreover, by the above argument $\psi(z)$ is real-valued on the lower boundary of the fundamental domain, taking on values in $[-64,0]$. The special value $\psi\left(-\frac{1}{2}+\frac{i}{2}\right)=-64$ arises from the relationships between $\psi(z)$ and $j(z)$ given by

$$
j(z)=\frac{(\psi(z)+256)^{3}}{\psi(z)^{2}}, \quad j(2 z)=\frac{(\psi(z)+16)^{3}}{\psi(z)} .
$$

By using the fact that $j\left(-\frac{1}{2}+\frac{i}{2}\right)=j(i)=1728$, it is easy to see that $\psi\left(-\frac{1}{2}+\frac{i}{2}\right)$ must be a common root of the polynomials $(x+256)^{3}-1728 x^{2}=(x+64)(x-512)^{2}$ and $(x+16)^{3}-$ $1728 x=(x-8)^{2}(x+64)$. 
Next, let

$$
F_{2}(z)=2 E_{2}(2 z)-E_{2}(z)=1+24 \sum_{n}\left(\sum_{d \mid n, d \text { odd }} d\right) q^{n}
$$

be the unique normalized holomorphic modular form of weight 2 and level 2. Here $E_{2}(z)$ is the weight 2 Eisenstein series $E_{2}(z)=1-24 \sum_{n=1}^{\infty} \sigma(n) q^{n}$. The form $F_{2}(z)$ has integer coefficients and a single zero at the elliptic point $-\frac{1}{2}+\frac{i}{2}$. This can be seen by noting that $F_{2}\left(\frac{z}{2 z+1}\right)=F_{2}(z+1)=F_{2}(z)$ at $z=-\frac{1}{2}+\frac{i}{2}$, so an application of the modular equation yields $-F_{2}(z)=F_{2}(z)$. Uniqueness comes from applying the valence formula for $\Gamma_{0}(2)$ found in, for instance, [7]. Additionally, we define the Eisenstein series $S_{4}(z) \in M_{4}(2)$ as

$$
S_{4}(z)=\frac{E_{4}(z)-E_{4}(2 z)}{240}=q+8 q^{2}+28 q^{3}+\cdots ;
$$

it is easily checked that $S_{4}$ has integral Fourier coefficients and vanishes at $\infty$. It does not vanish at the cusp at 0 , as the valence formula shows that there are no cusp forms of weight 4 and level 2.

We now use these forms to construct a basis for $M_{k}^{\sharp}(2)$. For general even weight $k$, we write $k=4 \ell+k^{\prime}$, where $k^{\prime} \in\{0,2\}$. A basis for $M_{k}^{\sharp}(2)$ is given by

$$
f_{k, n}^{(2)}(z)=q^{-n}+O\left(q^{\ell+1}\right),
$$

for all integers $n \geq-\ell$. We note that this is an extension of the basis given in [4] for $M_{k}(2)$; similar sequences of modular forms for many levels appear in [8]. The basis elements are constructed as follows. We first define $F_{0}=1$ and set $f_{k,-\ell}^{(2)}(z)=S_{4}^{\ell}(z) F_{k^{\prime}}(z)$. Next, for each $n>-\ell$ we define $f_{k, n}^{(2)}(z)$ inductively by multiplying $f_{k, n-1}^{(2)}(z)$ by $\psi(z)$ and subtracting off earlier basis elements. Note that since $S_{4}, F_{k^{\prime}}$, and $\psi$ have integral Fourier coefficients, each $f_{k, n}^{(2)}(z)$ has integral Fourier coefficients and is of the form $S_{4}^{\ell}(z) F_{k^{\prime}}(z) F(\psi(z))$, where $F(x)$ is a polynomial with integer coefficients of degree $n+\ell=n+\left\lfloor\frac{k}{4}\right\rfloor$. (In fact, $F(x)$ is a generalized Faber polynomial; see [9], [10].) Thus, if all of the zeros of the basis elements $f_{k, n}^{(2)}$ lie on the lower boundary of the fundamental domain, then all of the zeros of the polynomial $F(x)$ must lie in the interval $[-64,0]$.

The main result of this paper is the following theorem.

Theorem 1. Let $f_{k, n}^{(2)}(z)$ be as above. If $\ell \geq 0$ and $n \geq 14 \ell+8$, or if $\ell<0$ and $n \geq 15|\ell|+8$, then at least $\left\lfloor\frac{\sqrt{3}}{2} n+\frac{k}{6}\right\rfloor$ of the $n+\left\lfloor\frac{k}{4}\right\rfloor$ nontrivial zeros of $f_{k, n}^{(2)}(z)$ in the fundamental domain for $\Gamma_{0}(2)$ lie on the lower boundary of the fundamental domain.

We note that the bounds $n \geq 14 \ell+8$ and $n \geq 15|\ell|+8$ are not sharp, and that often many more of the zeros lie on the arc. In fact, for certain weights $k$ close to 0 , all of the zeros of all of the $f_{k, n}^{(2)}(z)$ are on the lower boundary of the fundamental domain. However, some restriction on $n$ in relation to $\ell$ is necessary, as there are also examples of $f_{k, n}^{(2)}(z)$ with zeros elsewhere. We discuss this further in Section 6. Additionally, we obtain similar results for a family of modular forms in $M_{k}^{\sharp}(2)$ whose coefficients are dual to the Fourier coefficients of $f_{k, n}^{(2)}(z)$ and for a basis for the space $M_{k}^{\sharp}(3)$, showing that many of the zeros of these modular forms in the appropriate fundamental domain lie on the lower boundary. 
The remainder of this paper proceeds as follows: in Section 3, we give a generating function for the basis elements $f_{k, n}^{(2)}(z)$ and approximate their values on the lower boundary of the fundamental domain by a trigonometric function. In Section 4 we bound the error term to show that most of the zeros lie on the appropriate arc. Section 5 gives technical details on bounds for the error, and in Section 6 we discuss extensions of the main theorem to other modular forms for $\Gamma_{0}(2)$ and to forms for $\Gamma_{0}(3)$.

\section{Generating FunCtions And integration}

In this section we use Cauchy's integral formula to relate the basis elements $f_{k, n}^{(2)}(z)$ to a trigonometric function. Letting $r=e^{2 \pi i \tau}$, a generating function for the basis elements $f_{k, n}^{(2)}(z)$ is given in El-Guindy's paper [8, Theorem 1.2] as

$$
\sum_{n=-\ell}^{\infty} f_{k, n}^{(2)}(z) r^{n}=\frac{\left(S_{4}^{\ell} F_{k^{\prime}}\right)(z)}{\left(S_{4}^{\ell} F_{k^{\prime}}\right)(\tau)} \frac{\psi(\tau) F_{2}(\tau)}{\psi(\tau)-\psi(z)}
$$

Multiply by $r^{-n-1}$ and integrate around $r=0$. Changing variables from $r$ to $\tau$ and noting that $\psi(\tau) F_{2}(\tau)=\frac{d}{d \tau} \psi(\tau)$, we obtain

$$
f_{k, n}^{(2)}(z)=\int_{-\frac{1}{2}+i A}^{\frac{1}{2}+i A} \frac{\left(S_{4}^{\ell} F_{k^{\prime}}\right)(z)}{\left(S_{4}^{\ell} F_{k^{\prime}}\right)(\tau)} \frac{\frac{d}{d \tau}(\psi(\tau)-\psi(z))}{\psi(\tau)-\psi(z)} \frac{e^{-2 \pi i n \tau}}{-2 \pi i} d \tau
$$

where $A$ is some real number larger than $\frac{1}{2}$. We move the contour downward, noting that there is a pole whenever $\psi(\tau)-\psi(z)$ is zero, which happens when $\tau$ is equivalent to $z$ under the action of $\Gamma_{0}(2)$. There are no other poles, since $S_{4}$ has no zeros in the upper half plane, and if $k^{\prime}=2$, the zero of $F_{2}(\tau)$ is canceled by the zero of $\frac{d}{d \tau} \psi(\tau)=\psi(\tau) F_{2}(\tau)$. The closed contour that is the difference between the old integral and the new integral is in the clockwise direction, so we get a factor of -1 in Cauchy's integral theorem, and at each pole, we obtain a term of $-2 \pi i$ times the residue of the integrand.

If a function $f(\tau)$ has a zero of order $n$ at $\tau_{0}$, its logarithmic derivative has a simple pole with residue $n$. In calculating the residue, note that part of the integrand is the logarithmic derivative of $\psi(\tau)-\psi(z)$, which has a simple zero exactly at the values we are looking at, since $\psi$ is a Hauptmodul for $\Gamma_{0}(2)$. This means that the logarithmic derivative of $\psi(\tau)-\psi(z)$ at points equivalent to $z$ under $\Gamma_{0}(2)$ will just give us a factor of 1 in the residue. Supposing that $\tau=\gamma z=\frac{a z+b}{c z+d}$ for some $\gamma \in \Gamma_{0}(2)$, this is then multiplied by

$$
\frac{e^{-2 \pi i n \gamma z}}{-2 \pi i} \frac{\left(S_{4}^{\ell} F_{k^{\prime}}\right)(z)}{\left(S_{4}^{\ell} F_{k^{\prime}}\right)(\gamma z)}
$$

Since the denominator is a modular form of weight $k$ on $\Gamma_{0}(2)$, we have $\left(S_{4}^{\ell} F_{k^{\prime}}\right)(\gamma z)=$ $(c z+d)^{k}\left(S_{4}^{\ell} F_{k^{\prime}}\right)(z)$ for $\gamma=\left(\begin{array}{ll}a & b \\ c & d\end{array}\right)$, and the residue becomes $\frac{1}{-2 \pi i}(c z+d)^{-k} e^{-2 \pi i n(\gamma z)}$.

Assume that $z$ is on the lower boundary of the fundamental domain for $\Gamma_{0}(2)$. The first two points where $\tau$ is equivalent to $z$ through which the contour moves are $\tau=z$ and $\tau=\frac{z}{2 z+1}$. Calculating the residues, we find that

$$
f_{k, n}^{(2)}(z)-e^{-2 \pi i n z}-(2 z+1)^{-k} e^{-2 \pi i n\left(\frac{z}{2 z+1}\right)}=\int_{C} \frac{\left(S_{4}^{\ell} F_{k^{\prime}}\right)(z)}{\left(S_{4}^{\ell} F_{k^{\prime}}\right)(\tau)} \frac{\frac{d}{d \tau}(\psi(\tau)-\psi(z))}{\psi(\tau)-\psi(z)} \frac{e^{-2 \pi i n \tau}}{-2 \pi i} d \tau
$$


where $C$ is a contour that moves from left to right across the fundamental domain and passes below the points $\tau=z$ and $\tau=\frac{z}{2 z+1}$ and above all other points equivalent to $z$ under the action of $\Gamma_{0}(2)$.

We write $z=-\frac{1}{2}+\frac{1}{2} e^{i \theta}$ for some $0 \leq \theta \leq \frac{\pi}{2}$, so that $\frac{z}{2 z+1}=\frac{1}{2}-\frac{1}{2} e^{-i \theta}$; then the quantity $e^{-2 \pi i n z}+(2 z+1)^{-k} e^{-2 \pi i n\left(\frac{z}{2 z+1}\right)}$ can be simplified to

$$
(-1)^{n} e^{-i k \theta / 2} e^{\pi n \sin \theta} 2 \cos \left(\frac{k \theta}{2}-\pi n \cos \theta\right) .
$$

Putting all of this together and multiplying through by $e^{i k \theta / 2} e^{-\pi n \sin \theta}$, we end up with

$$
\begin{gathered}
e^{i k \theta / 2} e^{-\pi n \sin \theta} f_{k, n}^{(2)}\left(-\frac{1}{2}+\frac{1}{2} e^{i \theta}\right)-(-1)^{n} 2 \cos \left(\frac{k \theta}{2}-\pi n \cos \theta\right)= \\
e^{i k \theta / 2} e^{-\pi n \sin \theta} \int_{C} \frac{\left(S_{4}^{\ell} F_{k^{\prime}}\right)(z)}{\left(S_{4}^{\ell} F_{k^{\prime}}\right)(\tau)} \frac{\psi(\tau) F_{2}(\tau)}{\psi(\tau)-\psi(z)} e^{-2 \pi i n \tau} d \tau .
\end{gathered}
$$

By the argument in Section 2, the left hand side is a real-valued function of $\theta$. We note that the cosine function takes on alternating values of \pm 2 whenever $\frac{k \theta}{2}-\pi n \cos \theta$ is equal to $m \pi$ for $m \in \mathbb{Z}$. Since this quantity moves from $-n \pi$ at $\theta=0$ to $\frac{k \pi}{4}$ at $\theta=\frac{\pi}{2}$, we know that there must be at least $n+1+\left\lfloor\frac{k}{4}\right\rfloor$ times that this happens. Thus, if we bound the integral term in absolute value by 2 , then by the Intermediate Value Theorem we must have at least $n+\left\lfloor\frac{k}{4}\right\rfloor$ zeros of the modular form on this arc.

The dimension of the space of holomorphic modular forms $M_{k}(2)$ is $\left\lfloor\frac{k}{4}\right\rfloor+1$, and we get at most $\left\lfloor\frac{k}{4}\right\rfloor$ zeros not at elliptic points for these forms by the general valence formula in [7]. (Note that $F_{2}(z)$ has a zero at $-\frac{1}{2}+\frac{i}{2}$, which is already on the arc in question, so $f_{k, n}^{(2)}$ has a trivial zero there if $k^{\prime}=2$.) Counting a pole of order $n$ at $\infty$ and no other poles gives us a total of $n+\left\lfloor\frac{k}{4}\right\rfloor$ zeros of the basis element $f_{k, n}^{(2)}(z) \in M_{k}^{\sharp}(2)$ whose locations are unknown. This argument proves that if the weighted modular form is close enough to the cosine function, then all of these zeros must be simple and must be on this arc on the lower boundary of the fundamental domain.

Unfortunately, it is difficult to move the contour down far enough to prove that all of the zeros are on this arc; as the weight $k$ or the order $n$ of the pole increases, the contour will need to get closer and closer to $\tau=0$ if $\theta$ is close to 0 . Recall that for every fixed value of $z$, we need to prove that the integral is bounded by 2 , after moving the contour below that value of $z$. As $z$ gets close to the real line, this becomes very difficult-either the contour is not straight and the integral is harder to estimate, as $\tau$ does not have a fixed imaginary part, or the contour must pass through more residues, adding additional terms to the equation.

We can still prove that the majority of the zeros do indeed lie on this arc by choosing a fixed height for the contour, estimating the value of the integral along that contour, and showing that its absolute value is bounded above by 2 . The goal then is to choose a contour low enough to capture as many zeros as possible, yet high enough to avoid additional residues and to avoid large values inside the integral. We choose $\tau=u+\frac{i}{5}$ for $|u| \leq \frac{1}{2}$, so that the contour has constant imaginary part $\frac{1}{5}$.

For this choice to work, we must also limit the range of $z$, so that our contour passes below $\tau=z$ and $\tau=\frac{z}{2 z+1}$ but above other images of $z$ under $\Gamma_{0}(2)$. If $z=-\frac{1}{2}+\frac{1}{2} e^{i \theta}$ for $\frac{\pi}{6} \leq \theta \leq \frac{\pi}{2}$, then $z$ has imaginary part $\geq \frac{1}{4}$, and a contour at a height of $\frac{1}{5}$ picks up residues at $\tau=z$ and $\tau=\frac{z}{2 z+1}$ but no other points equivalent to $z$ under the action of $\Gamma_{0}(2)$; the maximum 
possible imaginary part of such a point is $\frac{1}{6}$. In this case, the quantity $\left(\frac{k \theta}{2}-\pi n \cos \theta\right)$ inside the cosine function has the value $\frac{k \pi}{12}-\pi n \frac{\sqrt{3}}{2}$ at $\theta=\frac{\pi}{6}$ and the value $\frac{k \pi}{4}$ at $\theta=\frac{\pi}{2}$, and passes through at least $\left\lfloor\frac{k}{6}+n \frac{\sqrt{3}}{2}\right\rfloor$ multiples of $\pi$. Bounding the integral by 2 for the appropriate $f_{k, n}^{(2)}(z)$ will finish the proof of Theorem 1 .

\section{BOUNDing THE INTEGRAL}

In this section we bound

$$
e^{i k \theta / 2} e^{-\pi n \sin \theta} \int_{C} \frac{\left(S_{4}^{\ell} F_{k^{\prime}}\right)(z)}{\left(S_{4}^{\ell} F_{k^{\prime}}\right)(\tau)} \frac{\psi(\tau) F_{2}(\tau)}{\psi(\tau)-\psi(z)} e^{-2 \pi i n \tau} d \tau
$$

for the values $z=-\frac{1}{2}+\frac{1}{2} e^{i \theta}$ with $\theta \in\left[\frac{\pi}{6}, \frac{\pi}{2}\right]$ and $\tau=u+\frac{i}{5}$ with $u \in\left[-\frac{1}{2}, \frac{1}{2}\right]$. We will also give some indication of how this bound might change if we allow $\theta$ to approach 0 and alter the countour accordingly. Details for the computation of the numerical bounds that appear here are provided in the next section.

We seek a bound for

$$
\begin{gathered}
e^{\frac{i k \theta}{2}} e^{-\pi n \sin \theta} f_{k, n}^{(2)}\left(-\frac{1}{2}+\frac{1}{2} e^{i \theta}\right)-(-1)^{n} 2 \cos \left(\frac{k \theta}{2}-\pi n \cos \theta\right)= \\
e^{\frac{i k \theta}{2}} e^{-\pi n \sin \theta} \int_{-\frac{1}{2}}^{\frac{1}{2}} \frac{\left(S_{4}^{\ell} F_{k^{\prime}}\right)\left(-\frac{1}{2}+\frac{1}{2} e^{i \theta}\right)}{\left(S_{4}^{\ell} F_{k^{\prime}}\right)\left(u+\frac{i}{5}\right)} \frac{\psi\left(u+\frac{i}{5}\right) F_{2}\left(u+\frac{i}{5}\right)}{\psi\left(u+\frac{i}{5}\right)-\psi\left(-\frac{1}{2}+\frac{1}{2} e^{i \theta}\right)} e^{-2 \pi i n u} e^{\frac{2 \pi n}{5}} d u,
\end{gathered}
$$

which is real-valued, by something less than 2 . In absolute value, this integral is

$$
e^{-\pi n\left(\sin \theta-\frac{2}{5}\right)}\left|\int_{-\frac{1}{2}}^{\frac{1}{2}} \frac{\left(S_{4}^{\ell} F_{k^{\prime}}\right)\left(-\frac{1}{2}+\frac{1}{2} e^{i \theta}\right)}{\left(S_{4}^{\ell} F_{k^{\prime}}\right)\left(u+\frac{i}{5}\right)} \frac{\psi\left(u+\frac{i}{5}\right) F_{2}\left(u+\frac{i}{5}\right)}{\psi\left(u+\frac{i}{5}\right)-\psi\left(-\frac{1}{2}+\frac{1}{2} e^{i \theta}\right)} e^{-2 \pi i n u} d u\right| .
$$

Consider the exponential term $e^{-\pi n\left(\sin \theta-\frac{2}{5}\right)}$. We have chosen $\Im(\tau)=\frac{1}{5}$, so that $\sin \theta-\frac{2}{5}>0$ for $\theta \in\left[\frac{\pi}{6}, \frac{\pi}{2}\right]$, and this term has exponential decay as $n \rightarrow \infty$; in this case $e^{-\pi\left(\sin \theta-\frac{2}{5}\right)}<$ .73041. If we find an upper bound for the absolute value of the integral, then for large enough $n$ the right hand side is indeed less than 2 , and we can apply the Intermediate Value Theorem as desired. It turns out that we can find a bound for the absolute value that removes the dependence on $n$, but may be exponential in $\ell$. However, if $n$ is large enough in relation to $\ell$, then we will see that for a fixed weight $k$, all but finitely many of the $f_{k, n}^{(2)}(z)$ have zeros on the appropriate arc.

We note that the absolute value of the integral is certainly bounded above by

$$
\int_{-\frac{1}{2}}^{\frac{1}{2}}\left|\frac{S_{4}\left(-\frac{1}{2}+\frac{1}{2} e^{i \theta}\right)}{S_{4}\left(u+\frac{i}{5}\right)}\right|^{\ell}\left|\frac{\left.F_{k^{\prime}}\left(-\frac{1}{2}+\frac{1}{2} e^{i \theta}\right) F_{2}\left(u+\frac{i}{5}\right)\right)}{F_{k^{\prime}}\left(u+\frac{i}{5}\right)}\right|\left|\frac{\psi\left(u+\frac{i}{5}\right)}{\psi\left(u+\frac{i}{5}\right)-\psi\left(-\frac{1}{2}+\frac{1}{2} e^{i \theta}\right)}\right| d u,
$$

and already the dependence on $n$ has vanished. For the terms involving $S_{4}$ and $F_{k^{\prime}}$, we find an upper bound for the maximum possible value of these terms over the appropriate ranges of $u$ and $\theta$, and pull these upper bounds outside of the integral. This leaves us with the contribution from

$$
\int_{-\frac{1}{2}}^{\frac{1}{2}}\left|\frac{\psi\left(u+\frac{i}{5}\right)}{\psi\left(u+\frac{i}{5}\right)-\psi\left(-\frac{1}{2}+\frac{1}{2} e^{i \theta}\right)}\right| d u
$$

which we consider in pieces. 
Consider first the quantity

$$
\left|\frac{S_{4}\left(-\frac{1}{2}+\frac{1}{2} e^{i \theta}\right)}{S_{4}\left(u+\frac{i}{5}\right)}\right|^{\ell} .
$$

Computations, explained in more detail in the next section, yield

$$
.014 \leq\left|S_{4}\left(u+\frac{i}{5}\right)\right| \leq 2.44141 .
$$

As a power series in $q$, we know that $S_{4}$ has positive coefficients, as seen in (2.1). The maximum value of $\left|S_{4}(\tau)\right|$ occurs when $u=0$, when $q$ is real and positive. Heuristically, the minimum value should occur when there is maximum cancelation between terms, or when $q$ is real and negative, so that $u= \pm \frac{1}{2}$, and we confirm this computationally. Decreasing $\Im(\tau)$ both increases the upper bound, as we are adding larger positive terms, and potentially decreases the lower bound due to cancelation.

Heuristically, the maximum value of $\left|S_{4}(z)\right|$ should occur when $(-1+\cos \theta)$ is close to 0 , meaning $\theta$ is close to 0 , as here $q$ is real, positive, and close to 1 . Similarly, the minimum value of $S_{4}(z)$ should occur when $(-1+\cos \theta)$ is close to \pm 1 , meaning $\theta$ is close to $\frac{\pi}{2}$; here $q$ is real but negative, so there is extensive cancelation when adding terms. In this case, though, the size of $q$ depends on $\theta$, as we have $\left|e^{2 \pi i\left(-\frac{1}{2}+\frac{1}{2} e^{i \theta}\right)}\right|=\left|e^{-\pi \sin (\theta)}\right|$. Computationally, the minimum indeed occurs at $\theta=\frac{\pi}{2}$, where $\left|e^{-\pi \sin \left(\frac{\pi}{2}\right)}\right| \approx .04322$. In general, we have

$$
\left|e^{2 \pi i\left(-\frac{1}{2}+\frac{1}{2} e^{i \theta}\right)}\right|=\left|e^{-\pi \sin (\theta)}\right| \leq e^{-\pi / 2} \approx .20788 .
$$

We compute that

$$
.03 \leq\left|S_{4}\left(-\frac{1}{2}+\frac{1}{2} e^{i \theta}\right)\right| \leq .99995 .
$$

Moving the lower bound on $\theta$ closer to 0 increases the maximum value of $\left|S_{4}(z)\right|$, though it does not appear to affect the minimum value.

Putting this together, we have, for $\ell \geq 0$,

$$
\left|\frac{S_{4}\left(-\frac{1}{2}+\frac{1}{2} e^{i \theta}\right)}{S_{4}\left(u+\frac{i}{5}\right)}\right|^{\ell} \leq|71.425|^{\ell},
$$

and for $\ell<0$,

$$
\left|\frac{S_{4}\left(-\frac{1}{2}+\frac{1}{2} e^{i \theta}\right)}{S_{4}\left(u+\frac{i}{5}\right)}\right|^{\ell} \leq|81.38034|^{|\ell|} .
$$

Next, we consider the term

$$
\left|\frac{F_{k^{\prime}}\left(-\frac{1}{2}+\frac{1}{2} e^{i \theta}\right) F_{2}\left(u+\frac{i}{5}\right)}{F_{k^{\prime}}\left(u+\frac{i}{5}\right)}\right| .
$$

If $k^{\prime}=2$, this is $\left|F_{2}\left(-\frac{1}{2}+\frac{1}{2} e^{i \theta}\right)\right|$, which is bounded above by 8.00067 . If $k^{\prime}=0$, this is $\left|F_{2}\left(u+\frac{i}{5}\right)\right|$, which is bounded above by 12.50005 . Either way, the contribution is no more than 12.50005. Note that $F_{2}(\tau)$ has positive coefficients, and is therefore large when $S_{4}(\tau)$ is large.

Finally, we consider

$$
\int_{-\frac{1}{2}}^{\frac{1}{2}}\left|\frac{\psi\left(u+\frac{i}{5}\right)}{\psi\left(u+\frac{i}{5}\right)-\psi\left(-\frac{1}{2}+\frac{1}{2} e^{i \theta}\right)}\right| d u .
$$


The Hauptmodul $\psi(z)$ is an injective mapping on the fundamental domain; it is real-valued and strictly decreasing on $\theta \in\left[0, \frac{\pi}{2}\right]$. We restrict ourselves to $\theta \in\left[\frac{\pi}{6}, \frac{\pi}{2}\right]$. Computation shows that $\psi(z) \in[-64,-.033]$ on this domain. In contrast, $\psi\left(u+\frac{i}{5}\right)$ takes on a wide range of values, including some with very large and some with very small modulus. Bounding the numerator and denominator separately yields a trivial upper bound of roughly 79000, while numerical calculations indicate that the actual maximum is a little larger than 1.

In order to achieve a sharper bound, we will instead consider a related quantity,

$$
D(z, \tau)=\frac{\psi(z)}{\psi(\tau)-\psi(z)}
$$

where we use $\psi(z)$ to indicate $\psi\left(-\frac{1}{2}+\frac{1}{2} e^{i \theta}\right)$ and $\psi(\tau)$ to indicate $\psi\left(u+\frac{i}{5}\right)$ for ease of notation. This quantity is related to our Hauptmodul expression by the identity

$$
\left|\frac{\psi(\tau)}{\psi(\tau)-\psi(z)}\right|=\left|1+\frac{\psi(z)}{\psi(\tau)-\psi(z)}\right|=|1+D(z, \tau)| .
$$

It is easier to work with $D(z, \tau)$, as we know the numerator is real valued and within a small range, and bounding this quantity proves useful in Section 6 when discussing extensions of Theorem 1 .

We will break our path of integration into pieces, and consider $\psi(\tau)$ in relation to $\psi(z)$ on each. As we know that $\psi(z)$ is real, we consider the real and imaginary parts of $\psi(\tau)=$ $\psi\left(u+\frac{i}{5}\right)$ separately for $u \in\left[-\frac{1}{2}, \frac{1}{2}\right]$. It is clear that $e^{2 \pi i \tau}=e^{-\frac{2 \pi}{5}}(\cos (2 \pi u)+i \sin (2 \pi u))$, and so $\Re\left(\psi\left(u+\frac{i}{5}\right)\right)=\Re\left(\psi\left(-u+\frac{i}{5}\right)\right)$, while $\Im\left(\psi\left(u+\frac{i}{5}\right)\right)=-\Im\left(\psi\left(-u+\frac{i}{5}\right)\right)$. With this in mind, we restrict our calculations to $u \in\left[-\frac{1}{2}, 0\right]$ and use symmetry for $u \in\left[0, \frac{1}{2}\right]$.

The numerical techniques described in the next section reveal that on the interval $u \in$ $[-.5,-.21516]$, we have either $\Re(\psi(\tau))>0, \Re(\psi(\tau))<-128$, or $|\Im(\psi(\tau))|>64$; it follows that $|D(z, \tau)|<1$ on this interval.

Next, we note that since $\psi(z)$ is real, then if we have the bound $\Im(\psi(\tau)) \geq A>0$, it follows that

$$
|D(z, \tau)| \leq\left|\frac{\psi(z)}{(\Re(\psi(\tau))-\psi(z))+A i}\right| .
$$

If $\Re(\psi(\tau)) \geq 0$, then this is bounded above by 1 , while if $\Re(\psi(\tau))<0$, then the maximum possible value of the right hand side for a fixed $\tau$ as $z$ varies is

$$
\sqrt{\left(\frac{\Re(\psi(\tau))}{A}\right)^{2}+1}
$$

occurring when $\psi(z)=\frac{A^{2}+\Re(\psi(\tau))^{2}}{\Re(\psi(\tau))}$. A lower bound for $\Re(\psi(\tau))$ thus gives us an upper bound for $|D(z, \tau)|$.

For $u \in[-.21516,-.18884]$ we have $\Im(\psi(\tau))>1$ and $\Re(\psi(\tau))>-.0175$. If $\Re(\psi(\tau)) \geq 0$, then our bound is 1 , and if $0>\Re(\psi(\tau))>-.0175$ then our bound is 1.00016. Either way, for $u \in[-.21516,-.18884]$, we have $|D(z, \tau)|<1.00016$. Similarly, on [-.18884, -.12878$]$ we have $\Im(\psi(\tau)) \geq .033$, and we obtain a bound of 1.13192 .

Finally, we consider $[-.12878,-0]$. We have $\Re(\psi(\tau))>-.01424$, and so

$$
|D(z, \tau)| \leq\left|\frac{\psi(z)}{-.01424-\psi(z)}\right|
$$

The maximum value occurs when $\psi(z)=-.033$, and we find $|D(z, \tau)|<1.75344$ here. 
Altogether, we have $|D(z, \tau)|<1.75344$. By breaking the integral into pieces, we compute more precisely that

$$
\int_{-\frac{1}{2}}^{\frac{1}{2}}|D(z, \tau)| d u \leq 2 \cdot 0.60496=1.20992 .
$$

The relationship in 4.2 allows us to conclude that

$$
\int_{-\frac{1}{2}}^{\frac{1}{2}}\left|\frac{\psi\left(u+\frac{i}{5}\right)}{\psi\left(u+\frac{i}{5}\right)-\psi\left(-\frac{1}{2}+\frac{1}{2} e^{i \theta}\right)}\right| d u<2.20992 .
$$

We can improve this further by noting that the fact that $\Re(\psi(\tau))>0$ on $[-.45787,-.22531]$ implies that the integrand is bounded by 1 here, yielding

$$
\int_{-\frac{1}{2}}^{\frac{1}{2}}\left|\frac{\psi\left(u+\frac{i}{5}\right)}{\psi\left(u+\frac{i}{5}\right)-\psi\left(-\frac{1}{2}+\frac{1}{2} e^{i \theta}\right)} d u\right|<1.74520 .
$$

We see that if we extend $\theta$ closer to 0 , and hence also decrease $\Im(\tau)$, this term has the most potential to blow up near $u=0$, as this is where $\psi(z)$ and $\Re(\psi(\tau))$ are both small. Additionally, if $0 \leq \theta \leq \frac{\pi}{2}$, then any contour with fixed imaginary part less than $\frac{1}{2}$, such as $\tau=u+\frac{i}{5}$, will cross the arc $z=-\frac{1}{2}+\frac{1}{2} e^{i \theta}$ and at least one of its images under $\Gamma_{0}(2)$, so restricting our $\theta$ values is necessary to avoid a zero in the denominator.

Putting all of these pieces together and using the fact that $\sin \theta$ is decreasing on $\left[\frac{\pi}{6}, \frac{\pi}{2}\right]$, we see that for $\ell \geq 0$,

$$
\begin{aligned}
& e^{-\pi n\left(\sin \theta-\frac{2}{5}\right)}\left|\int_{-\frac{1}{2}}^{\frac{1}{2}} \frac{\left(S_{4}^{\ell} F_{k^{\prime}}\right)\left(-\frac{1}{2}+\frac{1}{2} e^{i \theta}\right)}{\left(S_{4}^{\ell} F_{k^{\prime}}\right)\left(u+\frac{i}{5}\right)} \frac{\psi\left(u+\frac{i}{5}\right) F_{2}\left(u+\frac{i}{5}\right)}{\psi\left(u+\frac{i}{5}\right)-\psi\left(-\frac{1}{2}+\frac{1}{2} e^{i \theta}\right)} e^{-2 \pi i n u} d u\right| \\
& <.73041^{n}|71.425|^{\ell}(12.50005)(1.74520) .
\end{aligned}
$$

Note that $\left(.73041^{n}\right)(12.50005)(1.74520)<2$ if $n \geq 8$, and $\left(.73041^{n}\right) 71.425<1$ if $n \geq 14$; hence, the integral is less than our desired bound 2 if $\ell \geq 0$ and $n \geq 14 \ell+8$. Similarly, for $\ell<0$, we replace $|71.425|^{\ell}$ with $|81.38034|^{|\ell|}$, and find that our integral is bounded by 2 if $n \geq 15|\ell|+8$. We can then apply the Intermediate Value Theorem to prove that the appropriate number of zeros are on the desired arc.

\section{Rigorously COMPUting UPPER AND LOWER BOUNDS}

In the previous section, while bounding our integral we used upper and lower bounds on Eisenstein series and the Hauptmodul for values on a circular arc on the boundary of the fundamental domain and on a straight line segment. In this section we justify those bounds.

It is useful for most of these calculations to truncate each series. For a modular form $f$ with Fourier series $f=\sum a_{f}(n) q^{n}$, we will choose a positive integer $N$ and let $\tilde{f}$ be the truncation of the Fourier series of $f$ up to and including the $q^{N}$ term, and we let $R f=f-\tilde{f}$ be the remaining tail of the series. We bound $\tilde{f}$ and $R f$ separately.

The calculations for the Eisenstein series are straightforward, as we have explicit formulas for the Fourier coefficients in terms of divisor functions, while calculations for $\psi$ require a little more finesse. We do not have a nice formula or a sharp bound for the growth rate of the Hauptmodul coefficients, and they are quite large, so there are more terms making a significant contribution to the value of the series. We begin by bounding the values of the Eisenstein series, and then use those bounds to tame $\psi(z)$. 
By (2.1) we have

$$
S_{4}(z)=\sum_{n=1}^{\infty}\left(\sigma_{3}(n)-\sigma_{3}\left(\frac{n}{2}\right)\right) q^{n} .
$$

For $k \geq 1$, we can generously bound $\sigma_{k}(n)=\sum_{d \mid n} d^{k}$ by $\sqrt{n} \cdot \sqrt{n}^{k}+\sqrt{n} \cdot n^{k}$ by considering pairs of divisors $\left(d, \frac{n}{d}\right)$. Thus, $\sigma_{k}(n) \leq n^{\frac{k+1}{2}}+n^{k+1}$. If $\left|e^{2 \pi i z}\right| \leq t$, then we can bound $R S_{4}(z)$ by

$$
\begin{aligned}
\left|\sum_{n=N+1}^{\infty}\left(\sigma_{3}(n)-\sigma_{3}\left(\frac{n}{2}\right)\right) q^{n}\right| \leq & \sum_{n=N+1}^{n=N+10}\left(\sigma_{3}(n)-\sigma_{3}\left(\frac{n}{2}\right)\right) t^{n}+ \\
& \sum_{n=N+11}^{\infty}\left(n^{2}+n^{4}\right) t^{n}-\sum_{n=\left\lfloor\frac{N+11}{2}\right\rfloor}^{\infty}\left(1+n^{3}\right) t^{2 n} .
\end{aligned}
$$

The last term reflects a lower bound on the summand $\sigma_{3}\left(\frac{n}{2}\right)$ coming from the even terms. Standard Taylor series methods involving derivatives of the geometric series $(1-x)^{-1}=\sum x^{n}$ taken at $x=t$ allow us to bound each infinite series. For example,

$$
\sum_{n=N+11}^{\infty}\left(n^{2}+n^{4}\right) t^{n}=\frac{t^{2}+t}{(1-t)^{3}}+\frac{t^{4}+11 t^{3}+11 t^{2}+t}{(1-t)^{5}}-\sum_{n=1}^{N+10}\left(n^{2}+n^{4}\right) t^{n} .
$$

We take $N=50$. For $S_{4}(z)$, we note that $\left|e^{2 \pi i z}\right| \leq t=e^{-2 \pi \sin \left(\frac{\pi}{6}\right)}=e^{\frac{-2 \pi}{4}}$. We find that $\left|R S_{4}(z)\right| \leq 2.86404 \cdot 10^{-23}$.

To find an upper bound for $\left|S_{4}(z)\right|$, we explicitly compute $\sum_{n=1}^{50}\left(\sigma_{3}(n)-\sigma_{3}\left(\frac{n}{2}\right)\right) t^{n}$ with $t=e^{\frac{-2 \pi}{4}}$ and then add the tail. We find that $\left|S_{4}(z)\right| \leq .99995$ on the appropriate arc. For a lower bound on $\left|S_{4}(z)\right|$, we consider the real and imaginary parts of $S_{4}\left(-\frac{1}{2}+\frac{1}{2} e^{i \theta}\right)$ separately. We compute the first derivative with respect to $\theta$ for each part, and for $\theta \in\left[\frac{\pi}{6}, \frac{\pi}{2}\right]$, each derivative has a trivial upper bound of $\sum_{n=1}^{50} \pi n \cdot\left(\sigma_{3}(n)-\sigma_{3}\left(\frac{n}{2}\right)\right)\left(e^{-2 \pi / 4}\right)^{n}<8.01$. A computation reveals that either the real or the imaginary part of $S_{4}(z)$ is at least .0302 when $z=-\frac{1}{2}+\frac{1}{2} e^{i \theta}$ with $\theta=\pi\left(\frac{1}{6}+\frac{1}{3} \cdot \frac{n}{40000}\right)$, for all $0 \leq n \leq 40000$. The bounds on derivatives and on the tail $R S_{4}(z)$ limit how close $\left|S_{4}(z)\right|$ can get to 0 , and we therefore conclude that $\left|S_{4}(z)\right|$ is bounded below by .03 .

We can do similar upper bound calculations for $S_{4}(\tau), F_{2}(\tau)$, and $F_{2}(z)$, using the additional fact that $\left|e^{2 \pi i \tau}\right| \leq t=e^{-2 \pi / 5}$. For $F_{2}$, we bound the tail by

$$
\left|R F_{2}(\tau)\right|=\left|\sum_{n=N+1}^{\infty} a_{f}(n) q^{n}\right| \leq \sum_{n=N+1}^{n=N+10} a_{F_{2}}(n) t^{n}+24 \sum_{n=N+11}^{\infty}\left(\frac{n}{2}+n^{2}\right)\left(e^{-\frac{2 \pi}{5}}\right)^{n},
$$

where the use of $\frac{n}{2}$ instead of $n$ comes from the fact that we only consider odd divisors. We again take $N=50$ in each case, and find that $\left|S_{4}(\tau)\right| \leq 2.44141,\left|F_{2}(z)\right| \leq 8.00067$, and $\left|F_{2}(\tau)\right| \leq 12.50005$.

To compute a lower bound for $\left|S_{4}(\tau)\right|$, we trivially bound the derivatives of the real and imaginary parts of $S_{4}\left(u+\frac{i}{5}\right)$ by

$$
\sum_{n=1}^{50} 2 \pi n \cdot a_{S_{4}}(n)\left(e^{-2 \pi / 5}\right)^{n}<48.83
$$


We then compute the real and imaginary parts of $S_{4}\left(u+\frac{i}{5}\right)$ for $u=-\frac{1}{2}+\frac{1}{2} \cdot \frac{n}{123000}$, where $0 \leq n \leq 123000$, verifying that at least one of these values is larger than .014010. We conclude that $\left|S_{4}(\tau)\right|$ is bounded below by .014 on $u \in\left[-\frac{1}{2}, 0\right]$, and use symmetry to extend this to $\left[0, \frac{1}{2}\right]$.

We now consider the Hauptmodul $\psi(\tau)$. In the previous section we needed information about the size of the real and imaginary parts of $\psi(\tau)$ and the value of $\psi(z)$. For these computations, we work with the truncations $\tilde{\psi}(z)$ and $\tilde{\psi}(\tau)$, taking into account the growth of the real and imaginary parts of the truncations and the error caused by ignoring the tail. In this case, the trivial bound on the partial derivatives is much larger than in the Eisenstein series case, so we truncate each series up to and including the $q^{30}$ term to shorten our computation time.

We can express $\psi$ in terms of Eisenstein series of level 2 as

$$
\psi(z)=\frac{E_{4}(2 z)}{S_{4}(z)}-16
$$

we use this representation to bound $R \psi(z)$. Observe that if we truncate $\psi(z)$, then the tail satisfies

$$
\begin{aligned}
R \psi(z) & =\psi(z)-\tilde{\psi}(z) \\
& =\frac{E_{4}(2 z)}{S_{4}(z)}-16-\tilde{\psi}(z) \\
& =\left(\tilde{\psi}(z)+16+\frac{E_{4}(2 z)-(\tilde{\psi}(z)+16) \tilde{S}_{4}(z)}{\tilde{S}_{4}(z)}\right) \frac{\tilde{S}_{4}(z)}{S_{4}(z)}-16-\tilde{\psi}(z) \\
& \leq|\tilde{\psi}(z)+16|\left|\frac{\tilde{S}_{4}(z)}{\tilde{S}_{4}(z)-R S_{4}(z)}-1\right|+\frac{\left|\tilde{E}_{4}(2 z)-(\tilde{\psi}(z)+16) \tilde{S}_{4}(z)\right|+\left|R E_{4}(2 z)\right|}{\left|\tilde{S}_{4}(z)-R S_{4}(z)\right|} .
\end{aligned}
$$

We can now bound $R \psi(z)$. We compute bounds for the $q^{31}$ to $q^{40}$ terms directly to find that they contribute at most $6.46551 \cdot 10^{-8}$, and use the truncation formula to bound the remainder of the tail. In applying the formula, we truncate all Eisenstein series at $N=50$ to use the previously computed upper and lower bounds. Note that, working as before, we can use the fact that $E_{4}(2 z)=1+240 \sum_{n=1}^{25} \sigma_{3}(n) q^{2 n}$ to prove that $\left|R E_{4}(2 z)\right| \leq 6.40309 \cdot 10^{-29}$ and $\left|R E_{4}(2 \tau)\right| \leq 2.16794 \cdot 10^{-22}$. We compute bounds for $\tilde{\psi}(z)+16$ and $\tilde{E}_{4}(2 z)-(\tilde{\psi}(z)+16) \tilde{S}_{4}(z)$ by summing $\sum\left|a_{f}(n)\right| e^{\frac{-2 \pi n}{4}}$ for each series. We find that the bounds are 544.01429 and $7.29909 \cdot 10^{-13}$, respectively. Putting all of this together we have, for $N=30$,

$$
\begin{aligned}
|R \psi(z)| \leq & 6.46551 \cdot 10^{-8}+544.01429 \cdot\left(\frac{.03}{.03-7.05863 \cdot 10^{-30}}-1\right)+ \\
& \frac{7.29909 \cdot 10^{-13}+6.40309 \cdot 10^{-29}}{.03-7.05863 \cdot 10^{-30}} \\
< & 6.46754 \cdot 10^{-8}
\end{aligned}
$$


We repeat the same calculations for $\psi(\tau)$. We find that

$$
\begin{aligned}
|R \psi(\tau)| \leq & .001371+2593.07795 \cdot\left(\frac{.014}{.014-2.86404 \cdot 10^{-23}}-1\right)+ \\
& \frac{6.40510 \cdot 10^{-7}+2.16794 \cdot 10^{-22}}{.014-2.86404 \cdot 10^{-23}} \\
< & 0.00142 .
\end{aligned}
$$

Now that we have bounds on the error caused by truncation, we numerically compute that $\psi\left(-\frac{1}{2}+\frac{1}{2} e^{i \theta}\right) \leq-.033$ for $\theta \in\left[\frac{\pi}{6}, \frac{\pi}{2}\right]$ by calculating $\tilde{\psi}(z)$ for $N=30$ at $\theta=\frac{\pi}{6}$ and adding the bound for the tail $|R \psi(z)|$.

The bounds for $\psi(\tau)$ are slightly more difficult. As with lower bounds for the Eisenstein series, we consider the real and imaginary parts of $\psi(\tau)$ separately. The maximum possible growth rate for the real part is

$$
\left|\frac{d}{d u} \sum_{n=-1}^{30} a_{\psi}(n) e^{\frac{-2 \pi n}{5}} \cos (2 \pi n u)\right| \leq \sum_{n=-1}^{30} 2 \pi n \cdot\left|a_{\psi}(n)\right|\left(e^{\frac{-2 \pi}{5}}\right)^{n}<101197.78,
$$

and the same bound holds for the derivative of the imaginary part. We again compute values of the real and imaginary parts of $\psi(\tau)$ at a sampling of points, and use these bounds on the derivatives to find intervals over which the real and imaginary parts of $\psi(\tau)$ fall within the ranges given in the previous section.

\section{Extensions of TheOREM 1}

In this section we discuss the sharpness of Theorem 1, extend Theorem 1 to a dual family of weakly holomorphic modular forms for $\Gamma_{0}(2)$, and consider analogous theorems for families of modular forms for other genus zero subgroups.

We have shown that if $f_{k, n}^{(2)}(z)$ is a basis element for the space $M_{k}^{\sharp}(2)$ and $n$ is large enough compared to $|\ell|$, then the majority of zeros of $f_{k, n}^{(2)}(z)$ lie on the lower boundary of the fundamental domain for $\Gamma_{0}(2)$. We note that the bounds on $n$ are not sharp. For example, if $\ell>0$, then the $F_{2}\left(u+\frac{i}{5}\right)$ term in the numerator of the integrand takes on its largest values near $u=0$, while the $S_{4}\left(u+\frac{i}{5}\right)$ term in the denominator takes on its smallest values near $u= \pm \frac{1}{2}$, and we have simply taken absolute upper bounds for these functions on the interval $u \in\left[-\frac{1}{2}, \frac{1}{2}\right]$ without accounting for interaction between these terms. It is clear that sharper bounds on $n$ are possible, and it is natural to ask if the zeros of $f_{k, n}^{(2)}(z)$ always lie on $z=-\frac{1}{2}+\frac{1}{2} e^{i \theta}$. In fact, we now exhibit explicit examples for which the zeros are not on this arc.

Note that if $k^{\prime}=0$ and the degree $n+\ell=n+\frac{k}{4}$ of the Faber polynomial $F(x)$ is equal to 1 , then we can directly compute that $F(x)=x-(8 \ell-24)$. For the root of this polynomial to be in $[-64,0]$, we must have $-5 \leq \ell \leq 3$. Thus, if $\ell \geq 4$ or $\ell \leq-6$, the single nontrivial zero of the modular form $f_{k,-\ell+1}(z)$ will not be on the lower boundary of the fundamental domain. If $k^{\prime}=2$, the analogous polynomial is $x-8 \ell$, and we must have $-8 \leq \ell \leq 0$ for the zero to be on the lower boundary. Similarly, if $k^{\prime}=0$ and the degree $n+\ell$ of the polynomial $F(x)$ is equal to 2 , we obtain the polynomial $x^{2}+(48-8 \ell) x+\left(32 \ell^{2}-188 \ell+24\right)$, which has complex roots when $\ell<-6$ or $\ell \geq 6$. Such calculations can also be done for polynomials of 
other small degrees to find ranges of $\ell$ and $n$ which will not work. Thus, the theorem is not true in general, and some condition on the size of $n$ compared to $\ell$ is necessary.

Next, we consider a straightforward extension of Theorem 1 to obtain results for another family of modular forms for $\Gamma_{0}(2)$. Let $g_{k, n}^{(2)}(z)$, for $n \geq-\ell+1$, be the unique modular form in $M_{k}^{\sharp}(2)$ which vanishes at the cusp 0 and has Fourier expansion beginning

$$
g_{k, n}^{(2)}(z)=q^{-n}+O\left(q^{\ell}\right) .
$$

These $g_{k, n}^{(2)}(z)$ form a basis for the subspace of $M_{k}^{\sharp}(2)$ consisting of forms that vanish at 0 . It is straightforward to construct $g_{k, n}^{(2)}(z)$ by setting $g_{k,-\ell+1}^{(2)}(z)=S_{4}^{\ell} F_{k^{\prime}} \psi(z)$ and obtaining the $g_{k, n}^{(2)}$ for larger values of $n$ inductively by multiplying earlier basis elements by powers of $\psi(z)$ and subtracting the appropriate terms. These $g_{k, n}^{(2)}$ were studied in the $k=0$ case by Ahlgren [1], and generating functions for arbitrary even weight $k$ were found by El-Guindy [8]. We note their similarity to the $f_{k, n}^{(2)}(z)$. In fact,

$$
\sum_{n=-\ell+1}^{\infty} g_{k, n}^{(2)}(z) q^{n}=\frac{\left(S_{4}^{\ell} \psi F_{k^{\prime}}\right)(z)}{\left(S_{4}^{\ell} \psi F_{k^{\prime}}\right)(\tau)} \frac{\psi(\tau) F_{2}(\tau)}{\psi(\tau)-\psi(z)}=-\sum_{m \geq \ell} f_{2-k, m}^{(2)}(\tau) q^{m}
$$

and replacing $k$ with $2-k$ and switching $\tau$ and $z$ shows that the $m$ th Fourier coefficient of $f_{k, n}^{(2)}(z)$ is equal to the negative of the $n$th Fourier coefficient of $g_{2-k, m}^{(2)}(z)$.

With the above generating function, we can repeat the calculations in Section 3, replacing $f_{k, n}^{(2)}$ with $g_{k, n}^{(2)}$, to see that the quantity $e^{\frac{i k \theta}{2}} e^{-\pi n \sin \theta} g_{k, n}^{(2)}\left(-\frac{1}{2}+\frac{1}{2} e^{i \theta}\right)$ is real valued, and that its difference from $(-1)^{n} 2 \cos \left(\frac{k \theta}{2}-\pi n \cos \theta\right)$ is given by an integral almost identical to the integral for the $f_{k, n}^{(2)}(z)$, except that the $\psi(\tau)$ in the numerator of the integrand becomes a $\psi(z)$; hence our Hauptmodul expression is the $D(z, \tau)$ function defined in (4.1). Following the argument as before and using the bound for $\int|D(z, \tau)| d u$ in Section 4 , we find that if $\ell \geq 0$, the difference is at most $(.73041)^{n}(71.425)^{\ell}(12.50005)(1.20992)$, while if $\ell<0$, it is at most $(.73041)^{n}(81.38034)^{|\ell|}(12.50005)(1.20992)$. We obtain the following theorem.

Theorem 2. Let $g_{k, n}^{(2)}(z)$ be as above. If $\ell \geq 0$ and $n \geq 14 \ell+7$, or if $\ell<0$ and $n \geq 15|\ell|+7$, then at least $\left\lfloor\frac{k}{6}+n \frac{\sqrt{3}}{2}\right\rfloor$ of the $n+\left\lfloor\frac{k}{4}\right\rfloor-1$ nontrivial zeros of $g_{k, n}^{(2)}(z)$ in the fundamental domain for $\Gamma_{0}(2)$ lie on the lower boundary of the fundamental domain.

Finally, we consider extensions of this method to other subgroups. Consider $\Gamma_{0}(N)=$ $\left\{\left(\begin{array}{ll}a & b \\ c & d\end{array}\right) \in \mathrm{SL}_{2}(\mathbb{Z}): c \equiv 0(\bmod N)\right\}$. If $N=2,3,5,7,13$, then this is a genus zero subgroup with two inequivalent cusps and with Hauptmodul

$$
\psi_{p}(z)=\left(\frac{\eta(z)}{\eta(p z)}\right)^{\frac{24}{p-1}} .
$$

It is again possible to define analogous families $f_{k, n}^{(N)}(z)$ and $g_{k, n}^{(N)}(z)$ for these groups, and to use [8] to find a generating function for each family that mirrors (3.1). Moreover, given a modular form $f$ of level $N$ with real coefficients, we find that the quantity $e^{-\frac{i k \theta}{2}} f\left(-\frac{1}{N}+\frac{1}{N} e^{i \theta}\right)$ is real for $0 \leq \theta \leq \pi$; this is a natural place to look for zeros.

Our argument for the case $N=2$ relied on the ability to find a particular horizontal contour meeting certain criteria. If we can find a contour passing below the points $z=-\frac{1}{N}+\frac{1}{N} e^{i \theta}$ 
and $\frac{z}{N z+1}$ and above all other images of $z$ under the action of $\Gamma_{0}(N)$, then an application of Cauchy's residue theorem to the generating function shows that

$$
e^{\frac{i k \theta}{2}} e^{-\frac{2 \pi n \sin \theta}{N}} f_{k, n}^{(N)}\left(-\frac{1}{N}+\frac{1}{N} e^{i \theta}\right)-2 \cos \left(\frac{k \theta}{2}+\frac{2 \pi n}{N}-\frac{2 \pi n}{N} \cos \theta\right)
$$

is equal to $e^{\frac{i k \theta}{2}} e^{-\frac{2 \pi n \sin \theta}{N}}$ times the integral of the generating function along this contour. If we take the absolute value of the latter expression, then choosing a contour with horizontal height $A^{\prime}$ means we can pull $e^{-\pi n\left(\frac{2}{N} \sin \theta-2 A^{\prime}\right)}$ outside of the integral, and as long as $\frac{1}{N} \sin \theta>$ $A^{\prime}$, this term has exponential decay as $n$ grows. This means that any bound on the size of the remaining integral term proves a result for the location of some of the zeros of $f_{k, n}^{(N)}(z)$ for large enough $n$.

It is possible to find such a contour for the case $N=3$. We take a fundamental domain in the upper half plane defined by $\left\{z:-\frac{1}{2} \leq \Re(z)<\frac{1}{2}\right\} \cap\left\{z:\left|z-\frac{1}{3}\right|>\frac{1}{3}\right\} \cap\left\{z:\left|z+\frac{1}{3}\right| \geq \frac{1}{3}\right\}$.

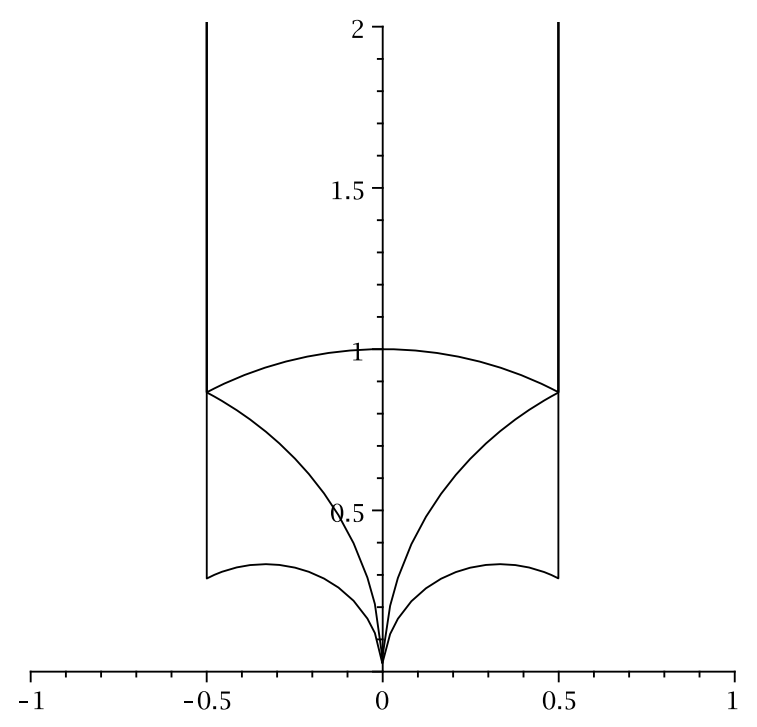

We let $k=6 \ell+k^{\prime}$ for $k^{\prime} \in\{0,2,4\}$, and for $n \geq-2 \ell-\left\lfloor\frac{k^{\prime}}{3}\right\rfloor$ we define a basis for $M_{k}^{\sharp}(3)$ by letting $f_{k, n}^{(3)}(z)$ be the unique element of $M_{k}^{\sharp}(3)$ with Fourier expansion beginning $q^{-n}+$ $O\left(q^{2 \ell+\left\lfloor\frac{k^{\prime}}{3}\right\rfloor+1}\right)$. We let $z=-\frac{1}{3}+\frac{1}{3} e^{i \theta}$, and require the height of the contour to be above $\frac{1}{9}$ to avoid any images of $z$ other than $\frac{z}{3 z+1}$. We restrict the range of $\theta$ values so that $z$ and $\frac{z}{3 z+1}$ lie above this contour. The contour may be as close to $\frac{1}{9}$ as we like, and we may allow $z$ to come as close to the contour as we like; once we fix these choices, the integral will have a finite upper bound, and the exponential term will eventually dominate when $n \geq C \ell$ for some constant $C$, so that the difference between the weighted modular form $f_{k, n}^{(3)}(z)$ and the cosine function is less than 2 . The number of zeros of $f_{k, n}^{(3)}(z)$ in the fundamental domain is $n+\left\lfloor\frac{k}{3}\right\rfloor$, and as the contour approaches a height of $\frac{1}{9}$ and $z$ is allowed to approach the contour, the number of zeros that can be proved to be on the lower boundary approaches $.9618 n+.2792 k$. Thus, we have the following theorem.

Theorem 3. Let $f_{k, n}^{(3)}(z)$ be as above. If $n$ is large enough compared to $\ell$, then the majority of the zeros of $f_{k, n}^{(3)}(z)$ in the fundamental domain for $\Gamma_{0}(3)$ lie on the lower boundary of the fundamental domain. 
A similar theorem clearly holds for the modular forms $g_{k, n}^{(3)}(z)$.

When $N=5,7$, or 13 , the shape of the fundamental domain is more complicated, and choosing an appropriate contour becomes correspondingly more difficult. We leave this as an open problem.

\section{REFERENCES}

[1] S. Ahlgren, The theta-operator and the divisors of modular forms on genus zero subgroups, Math. Res. Lett. 10 (2003), no. 5-6, 787-798.

[2] N. Andersen and P. Jenkins, Divisibility properties of coefficients of level p modular functions for genus zero primes, to appear in Proc. Amer. Math. Soc., arXiv:1106.1188v1 [math.NT].

[3] T. Asai, M. Kaneko, and H. Ninomiya, Zeros of certain modular functions and an application, Comment. Math. Univ. St. Paul. 46 (1997), no. 1, 93-101.

[4] D. Doud and P. Jenkins, p-adic properties of coefficients of weakly holomorphic modular forms, International Mathematics Research Notices 2010 (2010), 3184-3206.

[5] W. Duke and P. Jenkins, On the zeros and coefficients of certain weakly holomorphic modular forms, Pure Appl. Math. Q. 4 (2008), no. 4, 1327-1340.

[6] A. El Basraoui and A. Sebbar, Zeros of the Eisenstein series E $E_{2}$, Proc. Amer. Math. Soc. 138 (2010), no. $7,2289-2299$.

[7] A. El-Guindy, Linear congruences and relations on spaces of cusp forms, Int. J. Number Theory 3 (2007), no. 4, 529-539.

[8] _ Fourier expansions with modular form coefficients, Int. J. Number Theory 5 (2009), no. 8, 1433-1446.

[9] G. Faber, Über polynomische Entwicklungen, Math. Ann. 57 (1903), 389-408.

[10] _ Über polynomische Entwicklungen II, Math. Ann. 64 (1907), 116-135.

[11] S. Garthwaite, L. Long, H. Swisher, and S. Treneer, Zeros of some level 2 Eisenstein series, Proc. Amer. Math. Soc. 138 (2010), no. 2, 467-480.

[12] A. Ghosh and P. Sarnak, Real zeros of holomorphic Hecke cusp forms, Jour. Eur. Math. Soc. 14 (2012), no. $2,465-487$.

[13] H. Hahn, On zeros of Eisenstein series for genus zero Fuchsian groups, Proc. Amer. Math. Soc. 135 (2007), no. 8, 2391-2401 (electronic).

[14] R. Holowinsky and K. Soundararajan, Mass equidistribution for Hecke eigenforms, Ann. of Math. (2), 172 (2010), no. 2, 1517-1528.

[15] T. Miezaki, H. Nozaki, and J. Shigezumi, On the zeros of Eisenstein series for $\Gamma_{0}^{*}(2)$ and $\Gamma_{0}^{*}(3)$, J. Math. Soc. Japan 59 (2007), no. 3, 693-706.

[16] A. Miller and A. Pixton, Arithmetic traces of non-holomorphic modular invariants, Int. J. Number Theory 6 (2010), no. 1, 69-87.

[17] F. K. C. Rankin and H. P. F. Swinnerton-Dyer, On the zeros of Eisenstein series, Bull. London Math. Soc. 2 (1970), 169-170.

[18] R. A. Rankin, The zeros of Eisenstein series, Publ. Ramanujan Inst. No. 1 (1968/1969), 137-144.

[19] _ The zeros of certain Poincaré series, Compositio Math. 46 (1982), no. 3, 255-272.

[20] Z. Rudnick, On the asymptotic distribution of zeros of modular forms, Int. Math. Res. Not. (2005), no. 34, 2059-2074.

[21] J. Shigezumi, On the zeros of the Eisenstein series for $\Gamma_{0}^{*}(5)$ and $\Gamma_{0}^{*}(7)$, Kyushu J. Math. 61 (2007), no. $2,527-549$.

[22] _ On the zeros of certain Poincaré series for $\Gamma_{0}^{*}(2)$ and $\Gamma_{0}^{*}(3)$, Osaka J. Math. 47 (2010), no. 2, 487-505.

[23] K. Wohlfahrt, Über die Nullstellen einiger Eisensteinreihen, Math. Nachr. 26 (1963/1964), 381-383.

Department of Mathematics, Bucknell University, Lewisburg, PA 17870

E-mail address: Sharon.Garthwaite@bucknell.edu

Department of Mathematics, Brigham Young University, Provo, Ut 84602

E-mail address: jenkins@math. byu.edu 